\title{
Synthesis of $\mathrm{Tb}_{4} \mathrm{O}_{7}$ complexed with reduced graphene oxide for Rhodamine-B absorption
}

\author{
Hui Gao ${ }^{\text {a,* }}$, Yang Zhou ${ }^{\text {a }}$, Keqin Chen ${ }^{a}$, Xiaolong Li $^{\text {b* }}$
}

a School of Physical Science and Technology, Key Laboratory for Magnetism and Magnetic Materials of Ministry of Education, Lanzhou University, Lanzhou 730000, P.R. China

b Shanghai Synchrotron Radiation Facility, Shanghai Institute of Applied Physics, Chinese Academy of Sciences, Shanghai 201204, P. R. China

*Corresponding authors: hope@1zu.edu.cn (H. Gao)

lixiaolong@sinap.ac.cn (X.L. Li)

Tel.: +86-18993181143, Fax: +86-931-8913554

\begin{abstract}
Tb}_{4} \mathrm{O}_{7}$ complexed with reduced graphene oxide composite (Tb-rGO) had been designed and fabricated by a facile thermal reduction method. The formation of $\mathrm{Tb}_{4} \mathrm{O}_{7}$ particles and reduction of graphene oxide (GO) occurred simultaneously, and partial terbium ions would be complexed with rGO via oxygen-containing function groups on rGO sheets. Introducing of terbium ions could effectively tune the photoluminescence properties of rGO, and the composite exhibited the typical green emission of terbium ions as well as the blue self-luminescence of graphene entered at $440 \mathrm{~nm}$. Moreover, Tb-rGO had demonstrated its high capability as an organic dye (Rhodamine-B) scavenger with high speed and efficiency. The findings showed the promising applications for large-scale removal of organic dye contaminants, especially in the field of water treatment.
\end{abstract}

Keywords: Carbon materials; Luminescence;

\section{Introduction}

Addressing the severe water pollution arising from organic dyes is a challenge task [1]. Graphene-based materials have attracted tremendous attentions due to their good absorption ability for organic solvent, oils and organic dyes [2]. The commonly-used precursor for graphene, graphene oxide (GO), has contained a 
series of reactive oxygen functional groups, which would be useful for the practical applications through chemical functionalizations [3]. The availability of oxygen functional groups allowed GO sheets to react with manifold materials to form functionally advanced hybrid macroassmblies with superior characteristics. These made GO to be a possible starting material for immobilization of a large number of substances, such as rare earth metals, drugs, and inorganic nanoparticles $[4,5]$.

Graphene combined with rare earth ions composites have been reported in very recent years. For example, europium $(\mathrm{Eu})$ ions complexed with $\mathrm{rGO}$ had been created for the environmental protection for high absorption for organic dyes [4]. Terbium-containing GO was reported to be used for monitoring the hypochlorite [6]. Rare earth-based compounds displayed sharp emission bands; large stokes shifts, and a wide range of lifetimes as well as good chemical stabilities, which could be good candidate for graphene composites. Combined with rare earth would not only make the graphene luminescent, but also modify the inner structure of graphene.

In this work, $\mathrm{Tb}_{4} \mathrm{O}_{7}$ complexed with reduced graphene oxide (Tb-rGO) composite was synthesized by a facile thermal reduction approach. The objectives of this study were: (1) to investigate the inner structure of Tb-rGO; (2) to investigate the photoluminescence (PL) of Tb-rGO; (3) to evaluate the adsorption ability of Tb-rGO for organic dye.

\section{Experimental section}

GO was prepared from flake graphite powder using the modified Hummers' method [7]. $\mathrm{Tb}\left(\mathrm{NO}_{3}\right)_{3}$ solution was obtained by dissolving $\mathrm{Tb}\left(\mathrm{NO}_{3}\right)_{3}$ powders in the deionized water. $\mathrm{Tb}\left(\mathrm{NO}_{3}\right)_{3}\left(15 \mathrm{ml}\right.$ of $\left.0.3 \mathrm{mg} \mathrm{ml}^{-1}\right)$ aqueous solution was slowly added into the GO solution (30 $\mathrm{ml}$ of $\left.2 \mathrm{mg} \mathrm{ml}^{-1}\right)$ and stirred for 20 minutes at room temperature to form the uniform system. After drying the water, the mixture was calcined at $500{ }^{\circ} \mathrm{C}$ under an $\mathrm{H}_{2} / \mathrm{Ar}$ (volume ratio 1:5) atmosphere with a flow rate of $3 \mathrm{ml} / \mathrm{h}$ for $30 \mathrm{~min}$. Then, the chamber was slowly cooled to room temperature and the products were collected. rGO sample was also prepared without $\mathrm{Tb}$ component at the same synthesizing condition.

The morphology was characterized by scanning electron microscopy (SEM, Hitachi, S-4800) and transmission electron microscopy (TEM, Tecnai G2, FEI Company) with X-ray energy dispersive 
spectrometry (EDS). Raman spectroscopy was recorded using a Renishaw InVia Raman microscope with 532 nm laser. X-ray photoelectron spectroscopy (XPS, PHI-5702, Physical Electronics) was performed using a monochromated Al-Ka irradiation. Fourier transform infrared spectrum (FTIR) was recorded on a Nicolet NEXUS 670 FTIR spectrometer. And the PL spectra were recorded using an FLS-920T fluorescence spectrophotometer equipped with a $450 \mathrm{~W}$ Xe light source and double excitation monochromators.

\section{Results and discussion}

SEM image of Tb-rGO was provided in Fig.1a. After the thermal reducing process, GO sheets were partially reduced to the folding and ripple rGO nanosheets, which naturally aggregated and stacked to multilayers. It was observed the pariticles grew on the surface of rGO nanosheets, and some of them were wrapped by the flexible rGO nanosheets. It is believed that during the reaction, the decomposing of $\operatorname{Tb}\left(\mathrm{NO}_{3}\right)_{3}$ to terbium oxide $\left(\mathrm{Tb}_{4} \mathrm{O}_{7}\right)$ and the reducing of GO occurred simultaneously. Some large particles dispersed in crumpled pieces of silk fabric could be also clearly observed in TEM image (Fig. 1b). Fig.1c provided more detailed structural information of Tb-rGO. The image showed a well-defined $\mathrm{Tb}_{4} \mathrm{O}_{7}$ particle with lattice spacing of $0.36 \mathrm{~nm}$, which could be assigned to the $d$-spacing in the $\left(\begin{array}{lll}1 & 1 & 1\end{array}\right)$ planes of $\mathrm{Tb}_{4} \mathrm{O}_{7}$ [8]. EDS spectrum (Fig. 1d) showed the distinctive existence of terbium in the composite.
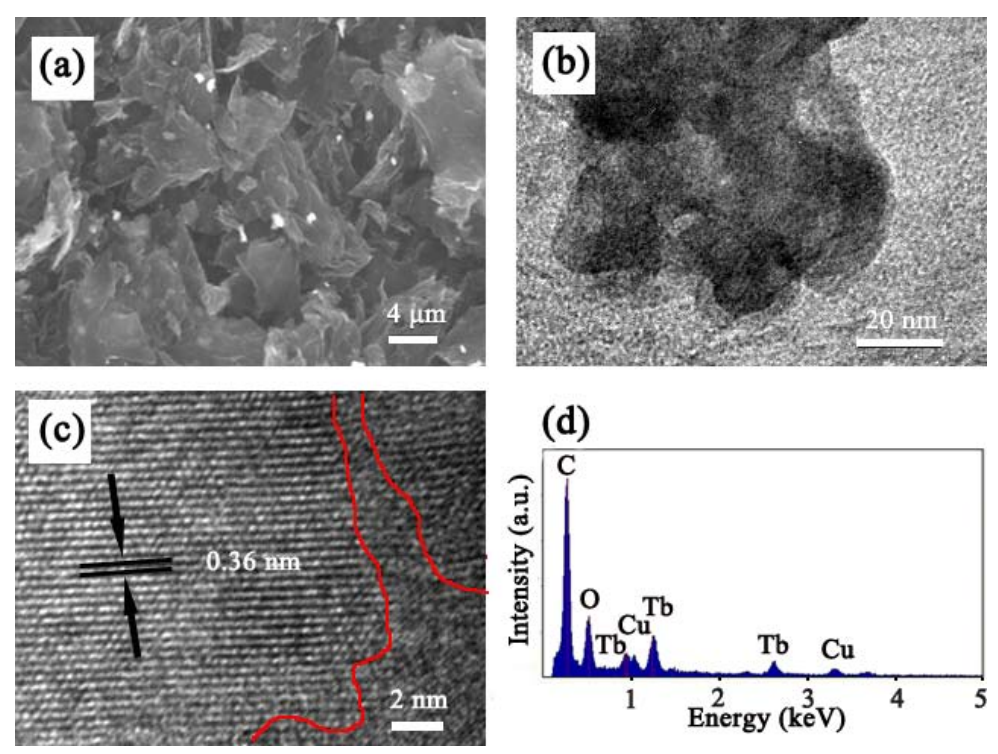

Fig. 1 The SEM image (a), TEM image (b), high resolution TEM (c) and EDS for Tb-rGO (d)

Raman spectrum for Tb-rGO was shown in Fig. S1, the D peak and G peak of Tb-rGO were located 
at $1368.4 \mathrm{~cm}^{-1}$ and $1595.5 \mathrm{~cm}^{-1}$. The $\mathrm{G}$ peak of Tb-rGO was noticed to have a red shift compared with the previous reported GO, implying not only the restacking of graphene sheets after the thermal reduction treatment, but also the modifying the coordination environment of the rGO nanosheets after complexed with terbium ions [9]. The existence of various oxygen functionalities (including the $\mathrm{C}=\mathrm{O}, \mathrm{C}-\mathrm{O}-\mathrm{C},-\mathrm{OH}$ and the Tb-O) would be attributed to the $\mathrm{D}$ peak. The $\mathrm{I}_{\mathrm{D}} / \mathrm{I}_{\mathrm{G}}$ ratio for Tb-rGO is 0.88 . And the presence of $2 \mathrm{D}$ peaks was also observed at $2935 \mathrm{~cm}^{-1}$, indicating the few-layered crystal structure of Tb-rGO. The FTIR spectrum for Tb-rGO was shown in Fig.S2. The peak at $3440 \mathrm{~cm}^{-1}$ could be attributed to the $\mathrm{O}-\mathrm{H}$ stretching vibration, and the unoxidized $\mathrm{sp}^{2} \mathrm{C}=\mathrm{C}$ bands located at $1550 \mathrm{~cm}^{-1}$, indicating the conversion from $\mathrm{GO}$ to $\mathrm{rGO}$. The absorption of $1060 \sim 1180 \mathrm{~cm}^{-1}$ and $1630 \mathrm{~cm}^{-1}$ could be assigned to the vibrations of $\mathrm{C}-\mathrm{O}$ and $\mathrm{C}=\mathrm{O}$ stretching vibrations. And the peak at $571 \mathrm{~cm}^{-1}$ would be attributed to Tb-O vibration [10]. From the above analysis, the remaining oxygen-containing groups would be effectively coordinated with partial terbium ions on the rGO sheets.

Fig.2a showed the XPS results for Tb-rGO, detecting the existences of the carbon $(\mathrm{C})$, oxygen $(\mathrm{O})$ and $\mathrm{Tb}$ elements. High resolution of C 1s peak (Fig. 2b) could be decomposed into three components at 284.5, 285.1, and $287.9 \mathrm{eV}$. The main peak at the binding energy of $284.5 \mathrm{eV}$ was assigned to $\mathrm{sp}^{2}$ hybridized $\mathrm{C}$ atoms in graphene while the higher binding energy confirmed the presence of O-rich groups: epoxy/hydroxyls (C-O, $285.1 \mathrm{eV}$ ) and carbonyl $(\mathrm{C}=\mathrm{O}, 287.9 \mathrm{eV})[11]$. The $\mathrm{O} 1 \mathrm{~s}$ peak (Fig. 2c) could be fitted with two components: the peak located at $530.7 \mathrm{eV}$ originated from the $\mathrm{Tb}-\mathrm{OH}$ bonds and the peak at $532.3 \mathrm{eV}$ involved various functional oxygen groups including $\mathrm{C}-\mathrm{OH}, \mathrm{C}=\mathrm{O}$ and $\mathrm{O}=\mathrm{C}-\mathrm{OH}[12,13]$. The $\mathrm{Tb} 4 \mathrm{~d}$ peak was shown in Fig.2d, The peak at $150.1 \mathrm{eV}$ was surely assigned to $\mathrm{Tb}$ (III) species. And the Tb (IV) species were suggested for the peaks at $156.9 \mathrm{eV}$ and $167.5 \mathrm{eV}$ [14]. The results indicated that the $\mathrm{Tb}$ ions could be in the mixed valence of $\mathrm{Tb}$ (III) and $\mathrm{Tb}(\mathrm{IV})$. 

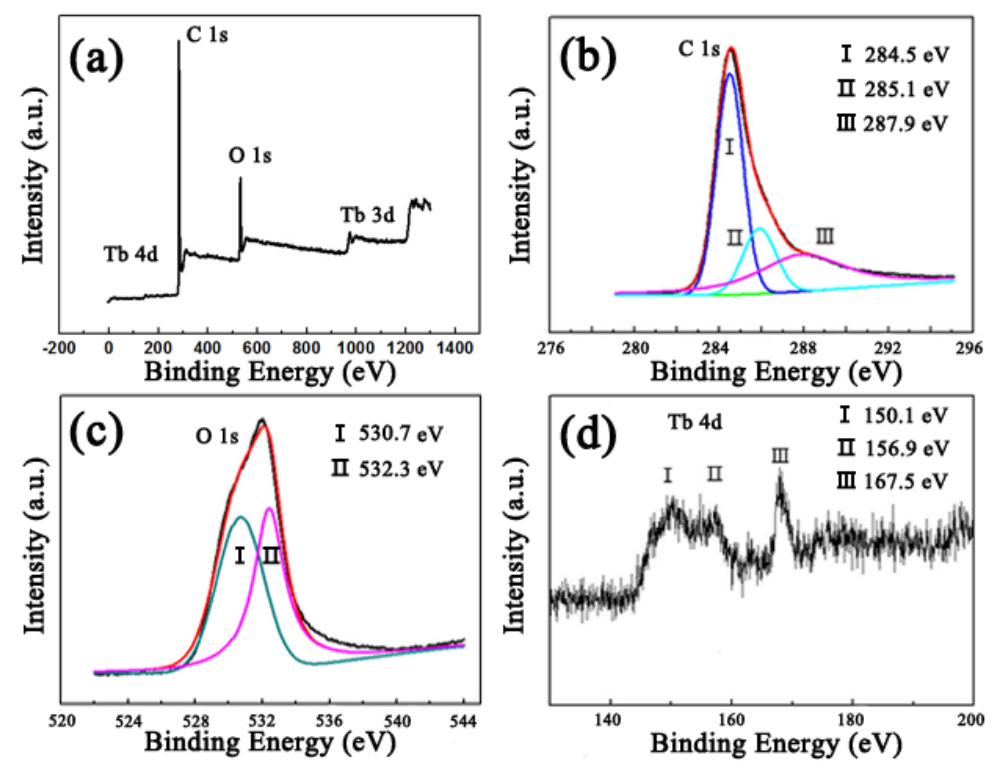

Fig. 2 (a) XPS survey spectrum of Tb-rGO. XPS high resolution spectra of C 1s (b), O 1s (c) and Tb 4d (d).

The emission and excitation spectra of the Tb-rGO were presented in Fig. 3a and b. A band ranging from 350 to $400 \mathrm{~nm}$ could be related to the direct excitation of the $\mathrm{f}-\mathrm{f}$ transition of the $\mathrm{Tb}$ ions. Compared with that of $\mathrm{Tb}$ ions in inorganic compounds, the excitation band seemed to be slightly broad, which was probably induced by the diversity of the transitions state of $\mathrm{Tb}$ ions in composite [15]. The emission peaks in the green region were noticed when using $378 \mathrm{~nm}$ as the excitation wavelength including ${ }^{5} \mathrm{D}_{4} \rightarrow{ }^{7} \mathrm{~F}_{6}(489 \mathrm{~nm}),{ }^{5} \mathrm{D}_{4} \rightarrow{ }^{7} \mathrm{~F}_{5}$ $(545 \mathrm{~nm}),{ }^{5} \mathrm{D}_{4} \rightarrow{ }^{7} \mathrm{~F}_{4}(580 \mathrm{~nm}),{ }^{5} \mathrm{D}_{4} \rightarrow{ }^{7} \mathrm{~F}_{3}(617 \mathrm{~nm})$ transitions, respectively. And the emissions of terbium ions were proposed not only from $\mathrm{Tb}_{4} \mathrm{O}_{7}$ particles but also from the terbium ions complexed with the rGO sheets. Our previous investigation for europium complexed with graphene self-assembly composite indicated that the introducing of europium ions would produce the newly formed $\mathrm{sp}^{2}$ clusters as well as the defects of the surfaces, and the ordered $\mathrm{sp}^{2}$ structure with small sizes $(1 \sim 3 \mathrm{~nm})$ isolated within the $\mathrm{sp}^{3} \mathrm{C}-\mathrm{O}$ matrix would generate the self-luminescence of graphene [16]. In the Tb-rGO system, the similar blue emission from grpahene itself was also observed in the range of $400-500 \mathrm{~nm}$. The PL results were in good agreement to the analysis of FTIR and Raman spectra.

Finally, we used Rhodamine-B (RhB) to investigate the absorption capacity of Tb-rGO. As a basic organic dye of the xanthene class, $\mathrm{RhB}$ is highly water soluble and widely used as a colorant in textiles and food stuffs. It is reported that the waste water with RhB is sincerely harmful to the environment, and it is important and urgent need to remove RhB in waste water. Dye adsorption experiment has been performed by 
mixing Tb-rGO solid powder $(10 \mathrm{mg})$ and $20 \mathrm{ml} \mathrm{RhB}$ solution $(2.5 \mathrm{ppm})$ at room temperature under natural light. The PL emission spectra and the corresponding digital photos of $\mathrm{RhB}$ solution as well as the $\mathrm{RhB}$ solution mixed with the Tb-rGO after 10 minutes and 1 hour were shown in Fig. 3c and 3d. As shown in Fig. 3d, the initial Rhodamine-B displayed a dark-pink color. After adding the Tb-rGO powders into the solution, the color almost disappeared in only $10 \mathrm{~min}$, and the RhB could be totally absorbed by the Tb-rGO in an hour. The PL emission spectra of the RhB solutions also supported the above phenomena. The fluorescence intensity (adding Tb-rGO) declined to $4 \%$ of the initial intensity presenting colorless after an hour, while for rGO, it remained $66 \%$ with visible hue after an hour(Fig.S3). As well known, RhB has rich aromatic rings, and the $\mathrm{rGO}$ would absorb dye molecules through $\pi-\pi$ stacking on the large specific surface area. After complexed with terbium oxide particles and terbium ions, more active sites would be introduced into the rGO, leading to the change of the microstructure of rGO (based on the analysis of Raman spectrum). Active terbium ions would be possible effectively bonded with the $-\mathrm{COOH}$ groups and $\mathrm{N}^{+}$cationic atoms existed in $\mathrm{RhB}$ due to their strong capability for coordination, resulting in effective enhancement of the absorption ability of the composite.
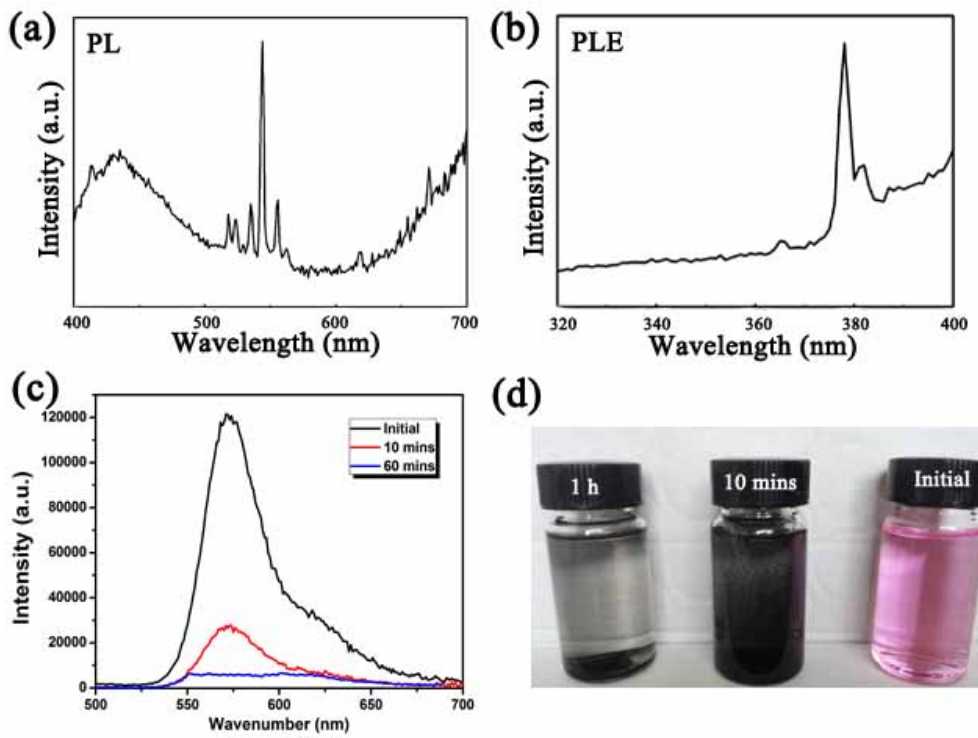

(d)

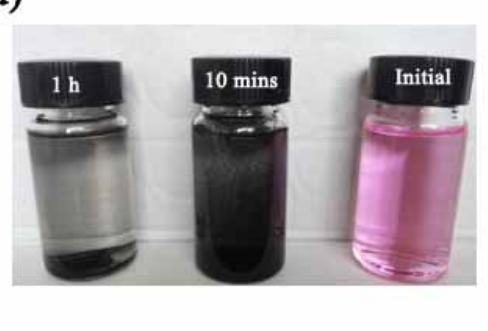

Fig. 3 PL emission (a) and excitation (b) spectrum of Tb-rGO. Emission spectra (under $365 \mathrm{~nm}$ exicitation) (c) and the corresponding digital photos (d) for initial RhB solution and the RhB solution mixed with Tb-rGO after10 minutes and 1 hour. 


\section{Conclusion}

In summary, the Tb-rGO composite was synthesized by the simple thermal reduction method. The analysis of Raman spectra, FTIR, XPS TEM and SEM convincingly certified that $\mathrm{Tb}_{4} \mathrm{O}_{7}$ particles as well as the terbium ions were well complexed with rGO sheets. The quenching of the fluorescence of organic dyes confirmed its potential applications in the field of environment protection.

\section{Acknowledgements}

This work was financially supported by the National Science Foundation of China $(51402140,11205235)$ and the National Basic Research Program of China (973 program, 2010CB934501).

\section{References}

[1] T. Madrakian, A. Afkhami, M. Ahmadi Spectrochimica Acta Part A, 99 (2012), pp. 102-109

[2] S. Lin, X. S. Zhao, Y. F. Li, K. Huang, R. X. Jia, C. Liang, et al Mater Lett, 132(2014),pp. 380-383

[3] X. L. Li, H. L. Wang, J. T. Robinson, H. Sanchez, G. Diankov, H.J. Dai J Am Chem Soc, 131(2009), pp. $15939-15944$

[4] D. Wang, H. Gao, E. Roze, K. Qu, W. Liu, Y. Shao, et.al J Mater Chem C, 1(2013),pp. 5772-5778

[5] X. Fan, G. Jiao, W. Zhao, P. Jin, X. Lin Nanoscale, 5(2013),pp. 1143-1152

[6] S. Pang, Z. Zhou, Q. Wang Carbon. 58(2013),pp. 232-237

[7] W. S. Hummers, R. E. Offeman J Am Chem Soc, 80(1958),pp. 1339-1339

[8] Y. P. Fang, A. W. Xu, L. P. You, R. Q. Song, J. C. Yu, H.X. Zhang, et al Adv Mater, 13(2003),pp. $955-960$

[9] B. K. Gupta, P. Thanikaivelan, T. N. Narayanan, L. Song, W. Gao, T. Hayashi Nano Lett, 11(2011),pp. $5227-5233$

[10] G. S. R. Raju, S. Buddhudu Mater lett, 62(2008),pp. 1259-1262

[11] R. Vercaemst, D. Poelman , L. Fiermans, R. L. Van Meirhaeghe, W. H. Laflere, F. Cardon J Electron Spectrosc Relat Phenom. 74(1995) pp.45-56

[12] Y. Wang, Y. Shao, D. W. Matson, J. H. Li, Y. Lin ACS Nano, 4(2010),pp. 1790-1798

[13] Y Sohn Ceram Int, 40(2014),pp. 13803-13811.

[14] K. Masumoto, C. Kimura, H. Aoki, T. Sugino Solid State Commun, 150(2010),pp. 1396-1399

[15] J. Sun, X. Zhang, Z. Xia, H. Du J Appl Phys, 111(2012),pp. 013101 
[16]Y. Dong, J. Shao, C. Chen, H. Li, R. Wang, Y. Chi, et al Carbon, 50(2012), pp. 4738-4743 